\title{
Poland syndrome: Two cases of new-borns with left-sided chest defect and dextrocardia.
}

\author{
Penka Petleshkova ${ }^{1,2^{*}}$, Maya Krasteva ${ }^{1,2}$, Desislava Gencheva ${ }^{2}$, Nevena Anesteva Ivanova ${ }^{2}$, Lilyana \\ Grozdanova $^{3}$, Nikoleta Parahuleva ${ }^{1,2}$, Anna Mihaylova ${ }^{4}$ \\ ${ }^{1}$ Department of Obstetrics and Gynecology, Medical University of Plovdiv, Plovdiv, Bulgaria \\ ${ }^{2}$ Department of Obstetrics and Gynecology, Neonatology, UMHAT 'St. George', Plovdiv, Bulgaria \\ ${ }^{3}$ Department of Medical Genetics, UMHAT 'St. George', Plovdiv, Bulgaria \\ ${ }^{4}$ Medical College, Medical University of Plovdiv, Bulgaria
}

\begin{abstract}
Poland Syndrome is a rare condition evident at birth that includes a wide range of malformations of chest and arm. The exact cause of the syndrome is unknown, but genetic involvement and a vascular abnormality of the subclavian artery are suspected. We report two cases of right-sided Poland Syndrome with true dextrocardia, diagnosed in neonatal period. Our report is focused on illustrating the importance of physical diagnosis and confirming that the associated features vary greatly among the individuals.
\end{abstract}

Keywords: New-born, Poland syndrome, Pectoralis muscle defect, Hand syndactyly, Dextrocardia.

Accepted on March 16, 2019

\section{Introduction}

Poland Syndrome (PS) is named after Alfred Poland, who first described the condition in 1841. The new syndrome was characterized by complete unilateral absence of M. Pectoralis major, M. Serratus anterior, and M. Obliquus externus abdominis with ipsilateral thoracic and upper limb defect [1]. PS is a rare congenital condition with an incidence between $1 / 20,000$ and 50,000 births worldwide as reported by different authors [2,3]. Most clinical cases are sporadic and familial patterns are rare. Recurrence is observed in about $10 \%$ of cases [3]. Commonly males are affected more than females and the right side of the body is involved mainly $[2,4,5]$.

\begin{abstract}
Aim
To describe two PS patients in order to present that the spectrum of defects includes a wide phenotypical variability, fluctuating clinical symptoms and outcome.
\end{abstract}

\section{Case Reports}

\section{Case 1}

A female baby born to primiparous 32 years old mother with uncomplicated pregnancy. No family history for genetic disorders or malformations. Birth in 41 weeks GA, with intrauterine growth restriction; vaginal delivery. No pathology of amniotic fluid, umbilical cord and placenta. Weight at birth-2480 g, height-48 cm. The baby was born with perinatal asphyxia, which impose intubation (APGAR at $1^{\text {st }}$ minute-2, $5^{\text {th }}$ minute-5). After initial resuscitation and intubation in the delivery room, patient is admitted in NICU.

Objective examination: Bad general condition, perioral and acrocyanosis, muscular hypotonia and hyporeflexia, flat chest on the left side as compared to the right; demonstrating respiratory distress-dyspnoea, weakened breathing in both chest sides, especially in left axilla. Hearth apex shifted to the right.

Malformative stigmas: underdevelopment of pectoralis minor muscle and the sternal part of pectoralis major muscle, deformation of the $2^{\text {nd }}, 3^{\text {rd }}$ and $4^{\text {th }}$ rib/visible sinking during inspiration; hypoplastic left palm, presence of transversal/ monkey/groove on the same side, brachysyndactyly of II, III and IV fingers (Figures 1A-1C).

Additional examinations: Chest X-ray deformation of the costal and cartilage parts of $3^{\text {rd }}, 4^{\text {th }}$ and $5^{\text {th }}$ rib, narrowed thirdfourth intercostal space, enlarged thymus in the right half.

Electrocardiography (ECG)-normal, Echocardiography (EchoCG), Patent foramen ovale (PFO), persistent pulmonary hypertension, dextroposition of the heart. Ultrasonography of the brain-hypoxic Ischemic Encephalopathy (HIE) $1^{\text {st }}$ grade. Ultrasonography of the abdomen-right position of the stomach.

Laboratory tests: Hypercapnic respiratory failure, positive blood culture-CONS and high values of C-reactive protein test 
(CRP). Cytogenetic test-karyotype 46, XX. Hearing testnormal.

Treatment: Mechanical ventilation-9 days, surfactant therapy, oxygen therapy-10 days, antibiotics, parenteral nutrition, catecholamines.

Clinical outcome: Discharged from the hospital in good general state at the age of 37 days. During the regular followup the child shows normal physical and neurological development. Plastic surgery of the hand is going to be performed. The chest defect will be corrected at early adolescence.
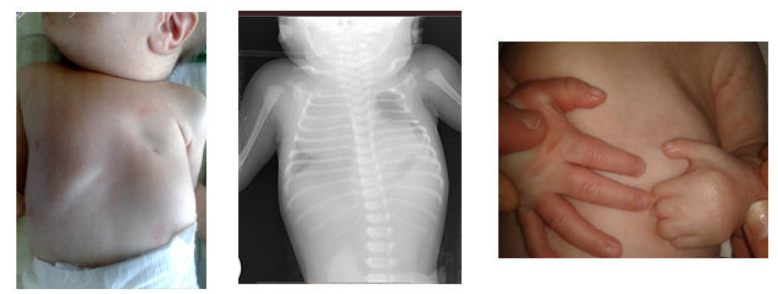

Figure 1. (A-C) Malformative stigmas observed in female baby (Case 1).

\section{Case 2}

A full term male baby born from a second uncomplicated pregnancy of 31 years old mother. No family history of congenital anomalies. Birth in $41^{\text {st }}$ weeks GA, vaginal delivery. No pathology of umbilical cord and placenta. Greenish amniotic fluid. Weight at birth-3840 g, height-51 cm. No data of perinatal asphyxia (APGAR at $1^{\text {st }}, 5^{\text {th }}$ minute-8). The baby is transported to NICU, demonstrating symptoms of impending respiratory failure, receiving mechanical ventilatory support during the transport.

On physical examination cyanosis is found, muscular hypotonia and hyporeflexia, flat chest on the left side, dyspnoea, weakened breathing in both chest sides. Hearth apex located on the right hemithorax.

Malformative signs: Underdevelopment of pectoralis minor muscle and the sternal part of pectoralis major muscle, malformative costal part of $3^{\text {rd }}$ and $4^{\text {th }}$ rib and underdeveloped end phalanx of the little finger of the left hand. Dysplastic, low-set ears (Figure 2A-2C).

Additional examinations: Chest and abdomen X-ray show respiratory distress syndrome; fourth intercostal space narrowed, dextrocardia and left position of the stomach.

ECG, EchoCG and ultrasonography of the brain-normal.

Laboratory tests: Respiratory acidaemia from blood gases test, positive blood culture-CONS and neutrophilia (86\%). Cytogenetic test-karyotype 46, XY. Hearing test-abnormal for both ears.

Treatment: Mechanical ventilation-12 hours, oxygen therapy-5 days, antibiotics.
Clinical outcome: Discharged from the hospital in good general condition at the age of 17 days. Parents are advised for regular follow-ups of their son. The chest defect is not going to be corrected. An additional hearing examination is planned.
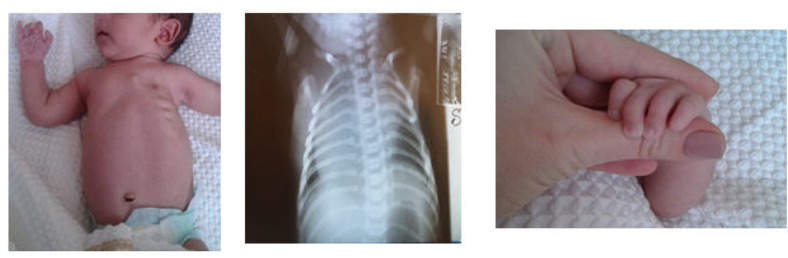

Figure 2. (A-C)Malformative stigmas observed in full term male baby (Case 2).

\section{Discussion}

Poland Syndrome is classically characterized by congenital absence (aplasia) of chest wall muscles on one side of the body unilateral underdevelopment or absence of pectoralis minor muscle and the sternal part of pectoralis major muscle. In some cases, there are associated skeletal abnormalities, that may include underdevelopment or absence of upper ribs and the bars of cartilage (costal cartilages) by which the ribs are attached to the sternum; abnormal elevation of the shoulder blade (Sprengel deformity); and/or shortening of the arm, with underdevelopment of the forearm bones (ulna and radius) [4]. Spine deformities are usually mild [3]. Most individuals with Poland Syndrome also have involvement of the hand on the affected side of the body- abnormally short, webbed fingers (synbrachydactyly) of the hand on the same side. Phalanges are underdeveloped or absent, resulting in abnormally short fingers (brachydactyly). In most cases, there is webbing (syndactyly) of certain fingers, particularly the index and middle fingers. Associated abnormalities may include underdevelopment or absence of the nipple and the darkened area around it (areola). In affected females there may be aplasia of the breast and deficiency of underlying tissues (subcutaneous fat) and abnormal patchy hair growth under the arm (axilla) [4].

This condition may be associated with several visceral anomalies which may include renal, cardiac, stomach, liver and biliary duct anomalies, central nervous system anomalies like encephalocele/exencephaly, microcephaly and other neural tube defects [4-6].

Some cases are reported as PS in association with Moebius syndrome, known as "Poland-Moebius Syndrome", presented with facial paralysis and chest wall defects and/or hand anomalies [7]. Additionally, it could be found foot deformity twisted out of shape or position facial and limb malformations, mild mental retardation and other abnormalities [4].

Associated platelet disorders, leukaemia, lymphomas [4,8] and congenital hyperinsulinism [2] have also been described.

Poland Syndrome affects the right side of the body more frequently than the left nearly 75 percent of cases. In 1998, researchers described a case with both sides of the body involved bilateral anomaly which includes absence of the left 
and the right pectoralis muscle, symmetric chest wall deformity, and bilateral hand involvement [4].

This anomaly is more common in boys than in girls and associated findings are highly variable [4,5]. The exact cause of the condition is unknown. Most of the cases are sporadic, with absence of family history. In some reported cases there is more than one family member affected- familial patterns of a parent and child or siblings born to unaffected parents $[4,9]$. One sibling may demonstrate all major symptoms of the condition, while the other sibling has only absence of pectoral muscle or hand involvement [9].

Some researchers suggest that the initial defect is damaged development of subclavian artery, vertebral artery and their branches or other mechanical factors leading to decreased or interrupted blood supply $[4,6,9]$ around $6^{\text {th }}$ week GA of embryonic development. The specific defect which is presented depends on the place and the rate of blood supply interruption, together with environmental factors. The syndrome is also known as Poland sequence, refers to a pattern of malformations derived from a single anomaly [4].

Recent hypothesis presume a genetic origin secondary to deleterious mutations of embryonic genes, which regulate development, and affect particularly chest wall muscles and skeleton structures [3].

Some authors suggest that significant enrichment of proteins involved in cell adhesion, blood coagulation, chondrogenesis, asymmetric development and skeletal muscle structure, are playing a role in PS development. One of the discussed genes is LAMA4. Lack of this gene caused haemorrhages associated with capillary defects in mice. He suggests that the deletion of LAMA4 induces haemorrhages during embryonic development and it may cause a variety of defects, possibly inducing those found in PS patients [10]. This would also be consistent with vascular hypothesis of PS [3].

The syndrome can be diagnosed prenatally using ultrasonography. The diagnosis is usually made at birth based upon typical phenotypic findings, a thorough clinical evaluation. The abnormality may be an incidental finding or found after specific examination- chest X-ray, chest computed tomography (CT scan), ECG and abdominal, brain and cardiac ultrasound scanning $[4,5]$.

Genetic counseling will be necessary for affected individuals and their families.

Treatment of PS is performed toward specific symptoms that each individual demonstrates. The functional disability is mild, so the patients usually present later for evaluation and discussion on aesthetic options [5]. Plastic surgery may be performed to rebuild the chest wall and to construct a breast mound in females; correction of skeletal abnormalities such as hand [3].

The cases we present, a male and a female new-born, are both from one and the same region with different ethnic background-case 2 is with gypsy origin. They are full-term new-borns, born from uncomplicated pregnancies, with no history for similar familial anomalies.

Both have the left side affected, which is evident finding at birth, as opposed to literature data where we found that the right part of the body is involved mainly [2,5].

Diagnosis of PS is based on characteristic physical findings, clinical evaluation and specialized tests. Phenotypically both patients demonstrate classic anomalies of chest muscles and bones, associated with hand deformities. Malformations of the girl case 1 are with more severe presentation than those of the boy, which accounts for the longer treatment time and stay in NICU after birth. Medical literature review shows that the condition may be linked with other visceral anomalies [5]. In both new-borns presented, the heart apex is on right side dextrocardia; and in case 1 the stomach is located on the right, without other abnormalities. We did not found any deviations from the normal karyotype in these cases.

Our patients are in bad general condition after delivery, with symptoms of respiratory distress syndrome- impending respiratory failure, due to combination of chest anomaly and presence of maternal- neonatal infection, cause of early neonatal CONS-sepsis with positive blood culture. Literature reports do not mention an association between Poland anomaly and maternal-neonatal infections.

\section{Conclusion}

Although rarely found, Poland Syndrome has variable phenotypic presentation, which is demonstrated right after childbirth. Knowing the condition allows early diagnosis, adequate treatment and follow-up during the neonatal period, as well as timely surgical correction.

\section{References}

1. Cingel V, Bohac M, Mestanova V, Zabojnikova L, Varga I. Poland syndrome: from embryological basis to plastic surgery. Surg Radiol Anat 2013; 35:639-646.

2. Giri D, Patil P, Hart R, Didi M, Senniappan S. Congenital hyperinsulinism and Poland syndrome in association with 10p13-14 duplication. Endocrinol Diabetes Metab Case Rep 2017; 1:16-125.

3. Romanini MV, Calevo MG, Puliti A, Vaccari C, Valle M, Senes F, Torre M. Poland Syndrome: A proposed classification system and perspectives on diagnosis and treatment. Semin Pediatr Surg 2018; 27:189-199.

4. Poland Syndrome. NORD (National Organization for Rare Disorders. 2007. https://rarediseases.org/rarediseases/poland-syndrome

5. Garg R, Saheer S, Gupta V, Mehra S. Poland sequence: Series of two cases and brief review of the literature. Ann Thorac Med 2012; 7:110-112.

6. Atasoy HI, Yavuz T, Altunrende S, Guven M, Kilicgun A, Polat O, Yesiller E, Duzenli S. A unique case of rightsided Poland syndrome with true dextrocardia and total situs inversus. Eur J Pediatr 2013; 172:269-272. 
7. Puvabanditsin S, Garrow E, Augustin G, Titapiwatanakul R, Kuniyoshi K.M. Poland-Möbius syndrome and cocaine abuse: a relook at vascular etiology. Pediatr Neurol 2005; 32:285-287.

8. Gerlinger I, Járai T, Lujber L, Pytel J. Poland's syndrome and head-and-neck tumour: an unusual association causing a reconstruction dilemma. Eur Arch Otorhinolaryngol 2007; 264:553-556.

9. Hennekam RC, Hofstee N. Familial liability to intrauterine vascular impairments. Pediatrics 1990; 86:326-327.

10. Thyboll J, Kortesmaa J, Cao R, Soininen R, Wang L, Iivanainen A, Sorokin L, Risling M, Cao Y, Tryggvason
K. Deletion of the laminin alpha4 chain leads to impaired microvessel maturation. Mol Cell Biol 2002; 22:1194-1202.

\section{*Correspondence to}

Penka Petleshkova

Department of Obstetrics and Gynecology

Medical University of Plovdiv

Plovdiv, Bulgaria 